\title{
Linx
}

Revue des linguistes de l'université Paris X Nanterre

$78 \mid 2019$

La linguistique des genres, en actes et en questions

\section{Genres et jeux de regards dans Trifles, pièce en un acte, et son adaptation en nouvelle 'A Jury of her Peers'}

Genres and visual interplay in Susan Glaspell's one-act play Trifles and adaption as a short-story, 'A Jury of Her Peers'

\section{Marie-Pierre Maechling}

\section{OpenEdition}

Journals

Édition électronique

URL : https://journals.openedition.org/linx/3071

DOI : 10.4000/linx.3071

ISSN : 2118-9692

Éditeur

Presses universitaires de Paris Nanterre

\section{Référence électronique}

Marie-Pierre Maechling, «Genres et jeux de regards dans Trifles, pièce en un acte, et son adaptation en nouvelle 'A Jury of her Peers' », Linx [En ligne], 78 | 2019, mis en ligne le 30 juin 2019, consulté le 26 juillet 2022. URL : http://journals.openedition.org/linx/3071 ; DOI : https://doi.org/10.4000/linx.3071

Ce document a été généré automatiquement le 26 juillet 2022.

Tous droits réservés 


\section{Genres et jeux de regards dans Trifles, pièce en un acte, et son adaptation en nouvelle 'A Jury of her Peers'}

Genres and visual interplay in Susan Glaspell's one-act play Trifles and adaption as a short-story, 'A Jury of Her Peers'

Marie-Pierre Maechling

\section{Introduction}

Susan Glaspell est un auteur américain du début du $20^{\mathrm{e}}$ siècle, à présent considérée comme pionnière pour ses positions féministes et dont les œuvres traitent souvent de problèmes de société tels que le rôle des femmes dans la société patriarcale, le sens de la démocratie américaine et les relations interpersonnelles (Gainor, $2001: 17$ ). Elle est l'auteur de plusieurs pièces, romans et nouvelles, et cet article se propose d'étudier ce qui est souvent cité comme sa pièce maîtresse : Trifles $^{1}$, pièce en un acte écrite en 1916, qu'elle a elle-même adaptée en nouvelle sous le titre « A Jury of her Peers » en 1917. Ces deux œuvres relatent l'entreprise simultanée de deux groupes distincts de personnages - les hommes de loi d'un côté et les femmes de l'autre - à la recherche d'indices ou d'un mobile permettant d'affirmer la culpabilité de Minnie Wright, accusée d'avoir tué son mari par strangulation.

La pièce Trifles est elle-même l'adaptation - ou directement inspirée - d'un procès que Glaspell couvrit du temps où elle était journaliste au DesMoines Daily News, procès intenté contre la femme d'un fermier accusée d'avoir assassiné son mari. Seule femme journaliste au procès, Glaspell tenta de comprendre le cas et, au fil de son enquête, elle prit de plus en plus causes et faits pour cette femme de 33 ans, mariée à un homme de 
60, dont elle décrit, dans un article au ton dramatique, l'existence, ou plutôt la nonexistence dans ce quotidien rural reclus.

Cette brève introduction au corpus suffit à comprendre son intérêt pour ce volume sur la linguistique des genres : elles-mêmes adaptées des chroniques judiciaires - un autre genre que nous n'étudierons pas ici - ces deux œuvres appartiennent à des champs génériques ${ }^{2}$ distincts et codifiés, à savoir le théâtre et le récit, dont le mode énonciatif et la structure sont facilement identifiables. En effet, le théâtre, ou plutôt le texte dramatique puisque c'est lui en tant que potentiel de performance et non sa performance réelle que nous allons étudier, se caractérise par un «système scripturaire » qui lui est propre, une "variation typographique » (Petitjean, $2009: 28$ ) entre les dialogues et les didascalies. Il est, de plus, caractérisé par une double énonciation. Dialogues et didascalies eux-mêmes ne sont pas exempts de faits linguistiques codifiés par le champ générique : à titre d'exemple pour les didascalies, on peut citer pour les langues française et anglaise, l'emploi du présent simple et d'énoncés non-finis (formes V-ING pour l'anglais, et toutes formes participiales pour le français), ou d'énoncés averbaux réduits à des monorhèmes ou des groupes nominaux ou adjectivaux. La pièce à l'étude n'y déroge pas :

(1) MRS HALE [Mildly.] ] Just pulling out a stitch or two that's not sewed very good.

[Threading a needle.] Bad sewing always made me fidgety. (Trifles : 31 )

La nouvelle, pour sa part, appartient en littérature au champ générique "récit ", "discours oral ou écrit qui assume la relation d'un événement ou d'une série d'événements » (Genette 2007, 14). Ce discours émane d'un narrateur adoptant le point de vue d'un centre focal, et propose une séquentialité qui n'est pas nécessairement chronologique. Temps, mode et voix sont autant de jeux à examiner dans le récit. Scripturalement, ces textes narratifs se caractérisent par une organisation en paragraphes, puis en chapitres ou parties, contrairement au texte de théatre divisé - ou divisible - en scènes et actes.

Les œuvres à l'étude s'inscrivent donc dans ces champs particuliers au sein desquels elles se différencient encore parce qu'elles sont des "formats courts", à savoir une pièce en un acte et une nouvelle. Mais parler de ces deux formes génériques requiert également une contextualisation socio-historique. Au tournant du $20^{\mathrm{e}}$ siècle aux ÉtatsUnis $^{4}$, la pièce en un acte divise: pour quelques-uns comme George Middelton qui écrivit "The Neglected One-Act Play» en 1912, elle manque grandement de considération; pour d'autres, comme l'éditeur Frank Shay, c'est un terrain expérimental, pratiqué, au même titre que la nouvelle, par des auteurs s'entrainant avant de passer à des formes plus longues. La pièce en un acte est en effet expérimentale, un vrai terrain de jeu pour les dramaturges comme Susan Glaspell, non encore réellement codifiée et prisée tant pour son économie et la tension qu'elle génère, que pour son coût bien moins élevé à produire. Elle divertit souvent un public plus populaire, moins fortuné, avant les grandes représentations de pièces traditionnelles.

La nouvelle, bien que largement considérée comme un art mineur notamment en raison de sa diffusion massive dans les magazines, est néanmoins pratiquée par des auteurs à succès depuis la fin du $19^{e}$ siècle et, sous l'impulsion d'Edgar Allan Poe, affiche des traits génériques tels que l'économie, le fonctionnement à partir de personnages types et antithétiques, la présence d'une pointe ou encore la marque d'une certaine oralité due au fait que le sujet approche l'anecdote, le fait divers, dont le « héros » est 
Monsieur-tout-le-monde ${ }^{5}$. L'on peut remarquer que ces critères, notamment la brièveté ou la différence de traitement des personnages, sont établis par rapport à un autre genre du champ narratif, le roman, puisque c'est aussi par comparaison que l'on différencie les genres discursifs au sein d'un même champ ${ }^{6}$.

Malgré ces différences indéniables de champ générique, les deux œuvres à l'étude sont textuellement très apparentées ${ }^{7}$ : on retrouve la pièce mot pour mot ou presque dans la nouvelle, une nouvelle extrêmement dialoguée (on n'y note aucune occurrence de discours transposé pour les échanges entre participants ni aucun résumé) qui présente la temporalité d'une "scène » dans la terminologie de Genette, ce choix de durée au plus fort potentiel mimétique ${ }^{8}$. Sauf pour l'incipit qui a été ajouté, la nouvelle présente la même unité de lieu, de temps et les mêmes personnages que sa sœur de théâtre. Et pour cause, l'intrigue se déroule dans la ferme isolée, ou plus exactement dans la cuisine de Minnie et John Wright : ce dernier a été étranglé, a priori par sa femme, et les hommes de loi cherchent un motif et des preuves tandis que les femmes sont censées récupérer des affaires pour l'épouse déjà amenée en prison. Nous sommes donc sur une scène de crime.

Dès lors s'invite un nouveau genre dans l'étude linguistique de notre corpus : celui de l'enquête (en anglais: detective fiction/clue puzzle tradition), enquête à la fois scientifique et psychologique puisque c'est un champ qui s'impose aux Etats-Unis à l'époque de l'écriture des œuvres. On peut noter la référence intertextuelle du titre de la pièce, "Trifles », qui rappelle une réplique de Sherlock Holmes dans la nouvelle de Conan Doyle "The Boscombe Valley Mystery " (Clausson, $2001: 85$ ). Ce genre discursif, s'il est alors plus apparenté au champ "récit » que théâtre, est déjà très codifié et le public, auditoire ou lectorat, en connaît les codes. Dans son article consacré au genre policier dans "A Jury of her Peers", Clausson (2001: 84) énumère les conventions thématiques en place au début du 20eme siècle. Parmi les 10 conventions qu'il recense, 7 se retrouvent dans nos deux œuvres au champs génériques différents :

1) Un meurtre énigmatique, dont on ne connait pas l'auteur

2) Un brillant détective (homme) professionnel ou amateur, potentiellement aidé

par un ami

3) Des indices apparemment sans importance qui se révèlent être essentiels

4) Une compétition ou un conflit entre le détective amateur ou professionnel et la

police chargée de l'enquête

5) L'usage de l'observation et de la déduction pour résoudre l'énigme

6) Le détective s'identifiant avec le criminel jusqu'au point de s'adonner lui-même à

des actes délictueux

7) La présence des personnages sur les lieux du crime ${ }^{9}$

À ces conventions, l'on peut ajouter que le genre "enquête policière " propose une " conception mimétique de la représentation » et "confronte le lecteur à l'actualité » (Evrard, 1996 : 91), traits définitoires que servent à la fois la temporalité du théâtre et de la nouvelle.

Les autres conventions (l'arrestation d'un suspect innocent et le dévoilement du nom du coupable, en général celui auquel on s'attendait le moins), si elles sont présentes, sont subverties pour les besoins de Glaspell dont les deux œuvres fonctionnent à deux niveaux : le premier, celui de l'intrigue et de sa résolution, et le deuxième, celui de la critique de la société patriarcale et de la difficile condition des femmes prisonnières de leur genre sexuel. Le titre Trifles joue sur les deux niveaux, tandis que «A Jury of her Peers ", lui, est politiquement plus affirmé en référent au fait que les femmes ne 
pouvaient siéger dans des jurys d'assises et n'étaient donc jugées que par le genre dominant, qui n'avait pas les clés pour appréhender la réalité de leur cas.

Le genre " enquête » étant commun à la pièce et à la nouvelle, et donc aux deux champs théâtre et récit, il se fait jour au travers de réalités linguistiques communes mais provoque aussi des réalisations différentes imposées par les deux champs génériques : nous avons choisi de nous concentrer sur les éléments relatifs au regard puisque ce dernier concerne diégétiquement le genre policier, narratologiquement, la nouvelle, et scéniquement, le texte dramatique. Dans un premier temps, nous étudierons les réalisations linguistiques inhérentes à l'histoire d'investigation, et nous examinerons ensuite les effets de point de vue dans les deux champs génériques, théâtre et récit, qui a priori s'opposent. Cette étude sera étroitement liée à la stratégie narrative des deux œuvres et à leur interprétation.

\section{Importance du regard dans le genre d'investigation}

Investiguer c'est chercher, et chercher c'est avant tout regarder, puis voir et enfin savoir. Dans les deux œuvres à l'étude, ce vocabulaire attendu du genre ainsi que les rôles des actants ne manquent pas à l'appel. On trouve ainsi le traditionnel échange entre enquêteur et témoin oculaire :

(2) COUNTY ATTORNEY Well, Mr Hale, tell just what happened when you came here yesterday morning.

$[\ldots]$

HALE I didn't hear or see anything (Trifles : 26)

(3) "Now, Mr Hale", he said in a sort of semi-official voice, "before we move things about, you tell Mr. Henderson just what it was you saw when you came here yesterday morning;" (" A Jury of her Peers», 82); "I didn't see or hear anything." ( A Jury of her Peers » : 84).

À la lecture, il est particulièrement frappant de voir l'emploi récurrent ${ }^{10}$ des lexèmes LOOK, SEE et également KNOW (dont nous parlerons plus tard) dans les deux œuvres ; leur répétition systématique dans l'appareil narratif (dans lequel je classerai les didascalies de la pièce et les parties narratives de la nouvelle encadrant le discours) se fait encore plus obsessionnelle dans la nouvelle qui, dans sa version originale, se conclut par une dernière occurrence (he didn't see her eyes) absente dans la pièce, jusqu'à apparaître de manière homonymique dans son titre, puisque le lexème peer appartient au champ lexical de la vision et s'insère tout à fait dans celui de l'investigation.

Le tableau 1 récapitule les emplois de LOOK et SEE, de loin les plus fréquents dans notre corpus:

Tableau 1 : Distribution de LOOK et SEE

\begin{tabular}{|c|c|c|c|c|}
\hline & \multicolumn{2}{|l|}{ Trifles } & \multicolumn{2}{|l|}{ «A Jury of her Peers » } \\
\hline LOOK (total) & 52 & & 75 & \\
\hline Look (agentif) & $\begin{array}{l}31 \\
\text { dans les } \\
\text { didascalies }\end{array}$ & 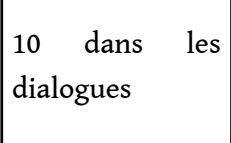 & $\begin{array}{l}51 \text { dans les passages } \\
\text { narratifs }\end{array}$ & 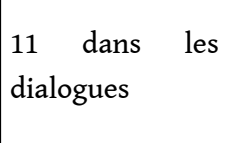 \\
\hline
\end{tabular}




\begin{tabular}{|c|c|c|c|c|}
\hline Look (attributif) & $\mid \begin{array}{lcl}2 & \text { dans } & \text { les } \\
\text { didascalies } & \end{array}$ & $\left|\begin{array}{lr}9 & \text { dans } \\
\text { dialogues } & \end{array}\right|$ & $\begin{array}{l}6 \text { dans les passages } \\
\text { narratifs }\end{array}$ & $\begin{array}{lr}7 & \text { dans } \\
\text { dialogues } & \text { les }\end{array}$ \\
\hline SEE (total) & 20 & & 43 & \\
\hline $\begin{array}{l}\text { See ("perceive with the } \\
\text { eyes") }\end{array}$ & $\begin{array}{l}1 \quad \text { dans } \quad \text { les } \\
\text { didascalies }\end{array}$ & 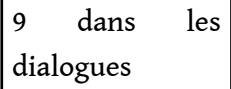 & $\begin{array}{l}17 \text { dans les passages } \\
\text { narratifs }\end{array}$ & 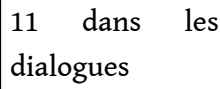 \\
\hline See (autres) & \multicolumn{2}{|c|}{10 (distribution peu pertinente) } & \multicolumn{2}{|c|}{15 (distribution peu pertinente) } \\
\hline
\end{tabular}

L'on constate d'un premier coup d'œil que ce vocabulaire a nettement augmenté dans la nouvelle et ce fait est notamment directement lié au changement de cible de Glaspell : un lectorat plus conservateur.

LOOK et SEE, s'ils ont en commun la notion de "vision », diffèrent dans leur sens, comme le fait remarquer Jean Chuquet (2003: 158). Le premier est agentif, avec un argument sujet "agent", alors que le deuxième est un verbe de perception involontaire et nécessite un expérient non-agent qui accueille la perception.

\subsection{Le geste " regarder »}

Dans Trifles, hommes et femmes regardent beaucoup car ils sont à la recherche d'indices. Le verbe LOOK n'apparait pas moins de 31 fois dans les didascalies, sans compter les autres lexèmes appartenant au même champ lexical (glance, examine, eyes falling on/ meeting/pointing to, etc.) qui constituent 13 autres occurrences du geste « regarder ». C'est donc l'élément narratif/descriptif des didascalies le plus fréquent, et l'on peut en dire de même dans «A Jury of her Peers ». Le tableau nous apprend aussi que, dans les deux œuvres, le verbe SEE est nettement moins employé, en particulier dans la pièce. Les personnages regardent donc plus qu'ils ne voient, et c'est une différence sémantique cruciale car la critique sociale de Glaspell joue précisément sur le fait que l'on peut regarder sans voir ${ }^{11}$. On note également que plus d'un tiers des emplois de SEE diffèrent du verbe de perception per se et de l'expérience de vision.

LOOK est ce que l'on appelle couramment un verbe d'action. Dans le dictionnaire unilingue, il est défini comme suit : to direct one's sight ; to give a certain direction to one's sight; to direct one's eyes upon some object or some portion of space ${ }^{12}$. La définition confirme bien l'agentivité du verbe : on fait une action intentionnelle, on fait donc un geste contrôlé. Dans la nouvelle au statut narratif, le verbe agentif LOOK apparaît 22 fois actualisé au prétérit et 4 fois à l'impératif dans les dialogues. Dans la pièce, il apparaît dans les didascalies 11 fois au présent et 12 fois à la forme V-ING, et 4 fois à l'impératif dans les dialogues.

Les prépositions ou adverbes directionnels qui y sont associées (about, around, at, in, etc.) confirment que ces regards sont de véritables mouvements voire déplacements du personnage/ comédien. En représentation, il doit donc utiliser son corps pour mettre ces jeux de regard en valeur et ce, afin d'atteindre deux buts - évidents - qui sont à reconstruire pour le lecteur du texte dramatique mais aussi de la nouvelle : 
- premièrement, représenter la quête du personnage, son attitude de détective - cette fonction est majoritairement assurée dans la pièce par les didascalies fonctionnelles qui contiennent les indications kinésiques :

(4) MRS PETERS [Looking in cupboard.] Why, here's a bird-cage. [Holds it up.] Did she have a bird, Mrs Hale? (31)

- deuxièmement, mettre en lumière les cibles de ce regard, afin d'alerter le spectateur/ lecteur ; cette fonction est à la fois assurée par les didascalies fonctionnelles et par le dialogue qui fait alors fonction de didascalie textuelle :

(5) MRS PETERS [Examining the cage.] Why, look at this door. It's broke. One hinge is pulled apart. (31)

Il se trouve en effet, comme l'indique la dernière partie de la définition citée, que le regard a un but, un objet au sens large, qui devient objet regardé et qui est donc mis en lumière par le caractère téléonomique du verbe. Les prépositions et adverbes qui accompagnent les verbes en disent long sur la sélection, le choix des objets regardés par les hommes et les femmes. De manière assez logique, c'est le verbe LOOK accompagné de l'adverbe directionnel ABOUT qui apparaît deux fois sur la première page ; les protagonistes prennent connaissance du lieu, de la cuisine, et ce balayage de la scène invite lecteur et spectateur à faire de même. Il y a tout au long de la pièce 9 occurrences de LOOK ABOUT et LOOK AROUND, sans cible particulière donc, ce qui est cohérent avec la recherche d'indices.

Mais la plupart du temps, LOOK est suivi d'une préposition (majoritairement AT, plus rarement TO/ON/IN) introduisant la cible du regard; autant de compléments d'objets ou compléments circonstanciels de lieu caractéristiques du genre d'investigation. Ces prépositions prennent un sens capital dans la diégèse, en incitant le spectateur/lecteur à poser également son regard sur ledit objet, mais elles entrent aussi dans le second niveau d'analyse des deux œuvres, à savoir la construction des personnages et la mise en regard hommes/femmes car l'objet regardé renseigne sur celui qui regarde. C'est ainsi que la distribution des verbes $L O O K$ et SEE doit être examinée pour entrer dans ce deuxième niveau d'analyse.

\section{2. $L O O K, S E E, K N O W$ : le genre d'investigation au service de la caractérisation sociale}

De manière assez significative, LOOK est le plus souvent suivi de AT + GN renvoyant à un objet, montrant les yeux du « regardant " pointés sur celui-ci ${ }^{13}$. La sélection des objets par chaque catégorie de personnages, ici les hommes et les femmes, en dit long sur leurs intuitions et leur compréhension de la tragédie qui s'est produite en ces lieux. Grâce aux didascalies, nous apprenons que les hommes portent leur regard sur le fauteuil à bascule, le mauvais placard et une étagère ; autrement dit, aucun des objets qu'ils sélectionnent ne peut les aider à comprendre la scène du crime ; ironiquement, lorsque que Mr Henderson remarque la cage à oiseau, élément clé du crime, c'est le lexème SEE qui est employé (seeing the birdcage, Trifles : 32); il n'est que récipiendaire de la vision, il n'a pas intentionnellement sélectionné l'objet, ce qui affaiblit la capacité d'investigation du personnage masculin. À l'inverse, les femmes sélectionnent tous les éléments importants à la compréhension du cas : les didascalies ou passages narratifs d'introduction indiquent qu'elles portent leur regard sur la miche de pain, le dessous de la table, l'ouvrage de couture de Minnie, la porte de la cage et le cou du petit oiseau 
mort. Elles se regardent également souvent l'une l'autre, signe de leur connivence croissante et de leur compréhension.

La distribution de SEE «vision» révèle que les hommes voient moins qu'ils ne regardent, et disent "ne pas voir» ou sont décrits comme tels. Cette vision, qui nécessite un récipiendaire individuel, est extrêmement liée au point de vue, un point de vue doté d'une critique du genre dans nos œuvres. La relation entre le verbe SEE et les hommes est décrite comme négative : ils ne voient pas, aveuglés par leur savoir genré ${ }^{14}$. Même s'ils dirigent leur regard vers un objet crucial, ils ne vont pas voir son importance, contrairement aux femmes. Cet aveuglement a été renforcé dans la nouvelle par l'ajout de la phrase finale He didn't see her eyes, qui conclut donc la pièce sur cette thématique.

L'emploi de SEE « vision » est rarement associé aux femmes ; quand il l'est, il est associé à une idée de malaise à la vue d'éléments troublants et de plus en plus significatifs. Fait remarquable, le lexème EYES, organe de la vision, est employé 27 fois dans « A Jury of her Peers " (contre 7 dans Trifles), et devient également symbole de compréhension les femmes sont de meilleurs détectives que les hommes - et de complicité entre les personnages féminins ; on rappelle que c'est le dernier mot de " A Jury of her Peers ».

Après $L O O K$ et $S E E$, il est frappant de voir la récurrence du verbe KNOW dans la pièce (41 occurrences) et dans la nouvelle (42). Sa distribution, elle aussi, en dit long sur le comportement du groupe homme et du groupe femme; son emploi en tant que savoir des uns et des autres révèle que les hommes, d'abord, l'emploient peu (9 fois) et surtout à l'affirmative (6 fois) : ils affirment savoir plus que ne pas savoir. Et l'une des répliques clés de «A Jury of her Peers ", qui a été rajoutée lors de l'adaptation, le fait apparaître :

(6) But would the women know a clue if they did come upon it?" he [Mr HALE] said.

(89)

Inversement, les femmes, qui l'emploient deux fois plus, affirment massivement leur ignorance dans leur quête, signe indéniable de la recherche, de la mise en problème (13 occurrences de KNOW + négation) contre seulement 4 emplois de KNOW affirmatif, et ces emplois sont très marqués : ils apparaissent tous les quatre dans le passage de la prise de conscience de Mrs Hale et surtout de Mrs Peters, prise de conscience contre laquelle Mrs Peters lutte jusqu'au bout grâce au verbe KNOW : elle chemine ainsi de We don't know who killed the bird à I know what stillness is (Trifles : 33), répété deux fois, et signe de son adhésion à la cause de Minnie.

En conclusion de cette étude du vocabulaire typique du genre d'investigation, nous comprenons que les éléments sans importance de la cuisine que les femmes, contrairement aux hommes, ont regardé, les font apparaître meilleurs détectives et meilleurs juges que les hommes de loi. Grâce à l'emploi du vocabulaire caractéristique du genre d'investigation, Glaspell a à la fois respecté deux conventions du genre qui veut que le détective ou l'apprenti détective soit plus fort que la police, et qu'il trouve la solution grâce à des indices apparemment de second ordre, mais elle a utilisé -voire subverti- le genre entier pour véhiculer un message politique anti patriarcal via la démonstration que les femmes sont de meilleures « lectrices » que les hommes grâce à leur savoir domestique, savoir qu'elle entend réhabiliter ${ }^{15}$. 


\section{Effets de points de vue dans les champs génériques}

Nous l'avons dit en introduction, les champs génériques narratif et théâtral diffèrent entre autres du point de vue énonciatif. Dans le premier, un narrateur adopte nécessairement un point de vue - auctoriel, actoriel et neutre selon Lintvelt (1981) tandis que dans le texte de théâtre, celui que l'on nomme plus souvent auteur que narrateur est à l'origine d'un texte dont la subjectivité ${ }^{16}$ (si elle est présente, ce qui est peu fréquent) n'est à cette époque qu'implicite. C'est précisément la grande différence qui existe entre les deux œuvres de Glaspell à l'étude ici : Trifles semble dénuée de toute marque de subjectivité tandis que la nouvelle adopte clairement le point de vue et donc la subjectivité d'une femme, Martha Hale. Pourtant, les deux œuvres entendent guider le lecteur vers le même point. Nous allons donc étudier ces effets de points de vue dans les deux champs génériques.

\subsection{L'exemple type de LOOK et SEEM}

Tableau 2. Distribution de LOOK et SEEM

\begin{tabular}{|c|c|c|c|c|}
\hline & \multicolumn{2}{|l|}{ TRIFLES } & \multicolumn{2}{|l|}{ «A Jury of her Peers » } \\
\hline \multirow[t]{2}{*}{ SEEM (total) } & \multicolumn{2}{|l|}{6} & \multicolumn{2}{|l|}{15} \\
\hline & 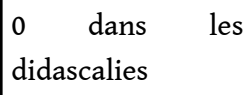 & $\begin{array}{lr}6 & \text { dans } \\
\text { dialogues } & \end{array}$ & $\begin{array}{l}9 \text { dans les passages } \\
\text { narratifs }\end{array}$ & $\begin{array}{ll}6 & \text { dans } \\
\text { dialogues } & \text { les }\end{array}$ \\
\hline \multirow[t]{2}{*}{$\begin{array}{l}\text { LOOK } \\
\text { (attributif) }\end{array}$} & \multicolumn{2}{|l|}{11} & \multicolumn{2}{|l|}{13} \\
\hline & $\begin{array}{lrl}2 & \text { dans } & \text { les } \\
\text { didascalies } & \end{array}$ & 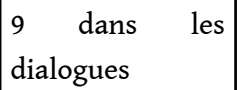 & $\begin{array}{l}6 \text { dans les passages } \\
\text { narratifs }\end{array}$ & $\begin{array}{ll}7 & \text { dans } \\
\text { dialogues } & \text { les }\end{array}$ \\
\hline
\end{tabular}

LOOK attributif et SEEM, tout en étant apparentés au genre d'investigation dans l'appréciation des apparences, sont deux verbes de perception caractéristiques de la différence de traitement du point de vue dans les deux champs génériques. Si dans les parties dialoguées, ils se réfèrent au point de vue des personnages et serviront donc plus le genre d'investigation, dans les parties «narratives» ils reflèteront le fonctionnement des champs génériques, comme nous le verrons dans un deuxième temps.

\subsubsection{Le point de vue des personnages dans les parties dialoguées}

LOOK attributif et SEEM ne traitent pas les apparences de la même façon; leur différence est proche de celle entre LOOK agentif et SEE. Avec LOOK, les apparences sont construites par un observateur qui en précise le canal sensoriel: celui-ci regarde un objet et déduit les apparences ; à nouveau, la téléonomie est un paramètre important dans l'emploi de LOOK attributif sauf que l'objet regardé devient l'argument sujet et que l'observateur reste implicite: ex: It (objet regardé) looked (copule) very lonesome (adjectif attribut) («A Jury of her Peers»: 82). La théorie de l'évidentialité, qui 
s'attache à « l'expression du mode d'accès à l'information énoncée » (Mélac 2014 : 31), étudie cette structure à sujet-percept et en démontre, selon les cas, son caractère perceptif visuel et factif ou bien inférentiel et non factif. Avec SEEM, le « canal sensoriel n'est pas précisé » (233), on n'est plus dans une perception nécessairement visuelle mais dans une "inférence plus mentale » (233). Un objet « donne l'impression » à un expérient, une impression émane de l'objet et est accueillie par le récipiendaire. Ce n'est pas le résultat construit d'une action/observation, c'est un sentiment. SEEM semble plus tourné vers l'appréciation, l'inférence subjective d'un locuteur, et en cela s'approche de la modalité épistémique qui évalue la probabilité d'une relation prédicative, tandis que LOOK, plus tourné vers l'objet regardé, est plus assertif (objectif ?) dans le sens où il dépend de l'observation, de la description.

Dans les parties dialoguées typiques du genre d'investigation, on voit très bien cette opposition entre l'observation et le sentiment dans plusieurs échanges: dans How did she look? She looked queer ("A Jury of her Peers »: 84), le shérif demande à Mr Hale de décrire ce qu'il a vu, tandis que dans How did she seem to feel about your coming? I don't think she minded ( A Jury of her Peers » : 85), il en appelle aux impressions de Mr Hale, à son avis personnel, d'où sa réponse grâce à un verbe d'opinion qui opère une modulation épistémique.

Si l'on considère toujours le genre d'investigation, l'emploi le plus remarquable de LOOK attributif est probablement son association à la locution AS IF, qui introduit un événement non réel, hypothétique et souligne le processus évidentiel inférentiel ; cette association n'est présente que deux fois dans le dialogue mais intervient au moment de la découverte des deux objets clés de l'intrigue par les femmes, à savoir l'ouvrage de couture de Minnie et la cage de l'oiseau :

(7) MRS HALE [Examining another block.] Mrs Peters, look at this one. Here, this is the one she was working on, and look at the sewing! All the rest of it has been so nice and even. And look at this! It's all over the place! Why, it looks as if she didn't know what she was about! (Trifles:30)

(8) MRS PETERS [Examining the cage.] Why, look at this door. It's broke. One hinge is pulled apart.

MRS HALE [Looking too.] Looks as if someone must have been rough with it. (Trifles : 31 )

Nous remarquons que l'emploi de $L O O K$ attributif est foncièrement dépendant de tous les autres verbes d'action LOOK et EXAMINE; ces derniers renforcent le processus observation-supposition et amoindrissent considérablement la part de subjectivité présente dans le point de vue énoncé par les femmes : c'est leur logique et leur bon sens qui ressortent de ces emplois. Encore un élément de caractérisation, puisque jamais cette locution n'est employée pour les hommes, soulignant un peu plus qu'ils sont incapables de formuler une hypothèse dans ce cas précis. ${ }^{17}$

Dans les dialogues, SEEM, lui, est majoritairement employé dans sa construction attributive et suivi de subjectivèmes (les lexèmes subjectifs dans la terminologie de Kerbrat-Orecchioni 1980) pas seulement évaluatifs mais également axiologiques et affectifs tels que cheerful («It never seemed a very cheerful place», Trifles: 28), mean ( "Seems mean to talk about her for not having things slicked up when she had to come away in such a hurry ", Trifles : 29), sneaking (« it seems kind of sneaking. Locking her up in town and then coming out here and trying to get her own house to turn against her! ", Trifles: 30), funny (" Seems funny to think of a bird here», Trifles: 31). Tous ces exemples, issus des répliques des personnages féminins, ont pour sujet IT désignant 
une situation générale (sauf le premier), donc un objet qui n'est pas directement et exclusivement observable par la vision, et dont la caractérisation repose sur un processus mental issu d'un ressenti. L'emploi quasi exclusif de SEEM par les femmes de la pièce ( 5 occurrences contre une seule pour les hommes) suggère la référence par l'auteur à leur sensibilité ignorée dans une société patriarcale. L'on peut également s'interroger sur l'emploi de SEEM dans les exemples ci-dessus, en particulier quand il est employé avec des lexèmes axiologiques dont le jugement est tourné vers les hommes ; car le message n'est-il pas réellement «It IS mean to talk about her for not having things up when she had to come away in such a hurry"?; ou encore "it IS sneaking. Locking her up in town and then coming out here and trying to get her own house to turn against her!»? SEEM affaiblit, bride une opinion marquée contre l'attitude des hommes, dans un processus d'autocensure grâce à ses liens à la modalité épistémique. La fiabilité de l'information est modalisée, non validée, pour, en réalité, en souligner la pertinence. Les jugements ou émotions que ces emplois induisent de la part de la source de perception féminine contribuent à leur caractérisation mais ont également un effet perlocutoire sur le public/le lectorat. Ils sont censés, à terme, contribuer à l'empathie recherchée par l'auteur ${ }^{18}$.

\subsection{2. $L O O K$ et SEEM dans les didascalies et passages narratifs}

L'implantation de la subjectivité est une toute autre affaire dans les parties non dialoguées des deux œuvres. Le tableau montre un grand contraste entre Trifles, où l'on note 2 occurrences de LOOK et aucune de SEEM, contre 15 occurrences au total dans « A Jury of her Peers ». Ce contraste ne nous étonnera pas, il suit la logique énonciative des deux champs génériques.

Le genre narratif ne se conçoit pas sans focalisateur. Comme beaucoup d'écrivains modernistes, Glaspell a choisi pour sa narration une perspective actorielle. Dans «A Jury of her Peers ", elle a favorisé un narrateur hétérodiégétique actoriel ${ }^{19}$, c'est-à-dire adoptant le point de vue du personnage féminin Martha Hales : les faits sont décrits à travers le regard de celle qui est désormais affichée comme le personnage principal de la nouvelle.

Dès le début de la nouvelle, le nom du centre focal est annoncé : il figure en première position dans la première phrase du long incipit - qui constitue l'ajout le plus significatif dans l'adaptation de Trifles - et dès la deuxième phrase, le lecteur est invité à suivre son œil :

(9) When Martha Hale opened the storm door and got a cut of the north wind, she ran back for her big woolen scarf. As she hurriedly wound that round her head her eye made a scandalized sweep of her kitchen [...] But what her eye took in was that her kitchen was in no shape for leaving: her bread all ready for mixing, half the flour sifted and half unsifted (81).

Comme avec LOOK agentif, le lecteur suit son regard et les cibles qu'il atteint : la cuisine et le pain, deux éléments également présents dans l'exposé liminaire de la pièce mais qui sont ici la cible d'un jugement féminin. Son point de vue est caractérisé : scandalized et in no shape for leaving montrent son souci pour les tâches domestiques. Cet ajout est évidemment de la plus haute importance car comme tout incipit, celui de «A Jury of her Peers » propose un programme de lecture.

Cette implantation du point de vue subjectif de Martha explique la présence de LOOK, et encore plus de SEEM dans les parties non dialoguées. Cette fois-ci, c'est la construction 
infinitive qui est la plus fréquente. Elle permet au narrateur de véhiculer la sensibilité, l'évaluation subjective et mentale de cette source du point de vue sur les situations auxquelles elle est confrontée :

(10) Mrs Gorman [...] had a voice that seemed to be backing up the law with every word (81)

(11) Sheriff Peters [...] leaned his hands on the kitchen table in a way that seemed to mark the beginning of official business. (82)

(12) The county attorney seemed suddenly to remember his manners. (87)

Grâce à cette perspective actorielle et au jeu entre SEEM et LOOK, Martha apparaît à la fois comme sensible et douée de bon sens et de raison.

Mais ces parties narratives qui font état de la subjectivité du personnage féminin n'existent évidemment pas dans la pièce, dont les didascalies affichent l'objectivité traditionnelle de l'époque. Susan Glaspell était d'ailleurs bien consciente de ce trait générique, et a longuement travaillé pour véhiculer la bonne impression, le bon guidage. Dans ses écrits, Glaspell explique qu'elle s'est posée en tant que spectatrice pour écrire sa pièce Trifles : elle se rendait tout simplement devant la scène du théâtre et imaginait le tout du point de vue du spectateur; il fallait être le plus précis possible pour que sa volonté soit parfaitement incarnée par les metteurs en scène - voire les lecteurs - potentiels.

Cette objectivité des didascalies, dont nous allons parler à présent, explique l'absence totale de SEEM, trop connecté à une source de perception individuelle.

Dans son ouvrage Le texte de théâtre, Françoise Rullier Theuret (2003:8) parle des didascalies en ces termes :

Rien ne rattache le texte didascalique au je-ici-maintenant de son énonciateur: jamais de $1^{\text {ère }}$ personne ou d'éléments marqués par la subjectivité. Les didascalies tiennent à la fois du commentaire, de la narration et du mode d'emploi dont elles ont le ton neutre.

Les termes "subjectivité » et "ton neutre " renvoient à l'empreinte potentiellement laissée par le narrateur - si tant est qu'on admette qu'il y en ait un. Nous sommes partie du principe que les didascalies étaient objectives, mais ne seraient-elles pas un tant soit peu subjectives, puisque notre tableau fait état de deux occurrences de LOOK qui requiert un observateur? Peut-on voir dans Trifles des marques de la subjectivité de l'énonciateur ou un regard singulier posé sur la scène?

Dès la présentation liminaire de la pièce, des termes a priori subjectifs ou déictiques sont effectivement présents :

(13) Scene : The kitchen in the now abandoned farmhouse of John Wright, a gloomy kitchen, and left without having been put in order- unwashed pans under the sink, a loaf of bread outside the breadbox, a dish towel on the table - other signs of incompleted work. At the rear the outer door opens and the Sheriff comes in followed by the County Attorney and Hale. The Sheriff and Hale are men in middle life, the County Attorney is a young man; all are much bundled up and go at once to the stove. They are followed by the two women - the Sheriff's wife first; she is a slight wiry woman,[with] a thin nervous face. Mrs. Hale is larger and would ordinarily be called more comfortable looking, but she is disturbed now and looks fearfully about as she enters. The women have come in slowly and stand close together near the door.

Regardons tout d'abord, à la ligne 1 , l'occurrence de NOW, adverbe déictique référant généralement au temps de l'énonciateur. Très clairement ici, ce NOW se rapporte au temps de la diégèse, au temps de l'énoncé puisque comme le dit Rullier Theuret (2003: 
8) « [r]ien ne rattache le texte didascalique au je-ici-maintenant de son énonciateur ». Ce NOW se rapporte au présent du narré, l'énonciation feint la simultanéité avec le temps zéro des personnages et du lecteur. Pour Jung (1994:18), le narrateur adopte « le point de vue d'un spectateur présent à une représentation ». Cela rappelle la technique de Susan Glaspell.

Les autres éléments soulignés en gras sont des subjectivèmes : Young, thin, slight, wiry, in middle life ou comfortable looking sont des adjectifs évaluatifs. Pourtant, le regard porté ici à des fins descriptives est finalement relativement neutre car il catégorise, caractérise les personnages au lieu de véhiculer une opinion. Étudions de plus près l'adjectif comfortable looking, dérivé à nouveau de ce verbe LOOK ayant trait au regard, et une occurrence similaire page 30 : the women look abashed. Ici, LOOK est pris dans son sens attributif et le regardant est implicite. Notre argument est que le regard se veut ici générique et non spécifique, et l'emploi de LOOK renvoie à tous les observateurs et non à un en particulier. On peut s'en convaincre en regardant l'environnement de la première occurrence qui dénote une caractéristique permanente du sujet: Mrs. Hale is larger and would ordinarily be called more comfortable looking. L'absence d'observateur particulier, ou la dissimulation d'un regard spécifique, est appuyée par la voix passive be called qui omet l'agent et par l'adverbe ordinarily. C'est à une opinion générique que l'on renvoie. De même, dans The women look abashed, l'apparence décrite doit être observée et même remarquée par n'importe quel spectateur regardant la pièce. L'emploi de $L O O K$ à sujet-percept permet d'ailleurs ici de ne pas préciser l'expérienceur (Mélac 2014 : 179), la source de la perception, et même de suggérer une vision collective. En somme, le verbe LOOK qui accompagne ces termes subjectifs ne fait que renvoyer à un jeu d'acteur et indique au spectateur ce qu'il doit déduire, en bon observateur, des indices visuels, probablement physiques ici, qu'il a sous les yeux. Plus que "elles ont l'air déconcerté », le lecteur/spectateur doit déduire: elles SONT déconcertées et cela se voit. Il en va de même pour les nombreuses occurrences de AS IF dans les didascalies, qui en appellent au jeu d'acteur (ex : makes a move as if to finish work (Trifles: 30)). L'on voit donc que le regard de l'observateur spécifique/narrateur est totalement affaibli ici et qu'en effet, on retrouve le ton neutre du mode d'emploi. Les subjectivèmes sont très peu nombreux ailleurs dans les didascalies de la pièce, et ne peuvent pas faire conclure à l'imposition d'un regard particulier. Si l'on devait donc parler en termes de focalisation, elle serait soit neutre soit plus vraisemblablement auctorielle sans intrusion particulière du narrateur ${ }^{20}$. Par ailleurs, nous pouvons remarquer que les descriptions liminaires ne comportent pas de repérage relatif à un énonciateur, même en position de spectateur, du type "à droite ", "à gauche " mais plutôt ce que j'appellerai du repérage absolu to the stove, near the door ${ }^{21}$. Cela sert encore l'effet "générique", sans lien à un regard spécifique, que nous avons tenté de démontrer.

\subsection{BE+V-ING : source du regard et point de vue}

En langue anglaise, $\mathrm{BE}+\mathrm{V}-\mathrm{ING}$ est associée à l'aspect, autre manifestation du regard d'un énonciateur spécifique. Dans son article de 1999 intitulé «BE+-ING est-il un marqueur d'aspect?", Larreya avance que BE+-ING suggère une "vision focalisante » (137) mettant donc l'emphase sur « le temps de l'observation » (145) et nous ajouterons, par ricochet, sur l'observateur. 
Dans la nouvelle, le temps employé est le prétérit caractéristique des narrations ultérieures. En raison de la perspective actorielle, et même si le récit est au prétérit, les déictiques correspondent au présent du personnage centre focal. C'est ainsi que l'on pourra lire :

(14) It looked very lonesome this cold March morning/he was not with them now for the very good reason that those potatoes never got to town yesterday" (82).

Les déictiques THIS, NOW et YESTERDAY renvoient clairement au présent de la situation énonciative de Martha Hale, personnage de la diégèse, et permettent au lecteur d'évoluer dans le même temps diégétique voire de s'identifier au regard marqué du centre focal. Le narrateur, en quelque sorte, se distancie de la diégèse et laisse au personnage l'autonomie $\mathrm{du}$ " voir » et du « dire ».

De même, l'on peut régulièrement voir dans l'emploi de la forme BE+V-ING la marque claire de la source du regard, à savoir le centre focal Martha Hale :

(15) The sheriff's wife did not reply. The silence made Mrs. Hale turn round. Mrs.

Peters was examining the bird-cage. (95)

(16) [...] then she glanced around and saw that Mrs. Peters was watching her (90)

(17) It came into Mrs. Hale's mind that that rocker didn't look in the least like

Minnie Foster - the Minnie Foster of twenty years before. [...]

"How did she - look ?" the county attorney was inquiring.

"Well," said Hale, "she looked - queer." (84)

Dans les exemples (15) et (16), l'événement en BE+V-ING est précédé d'une action au prétérit simple qui redirige le regard de Mrs Hale sur la situation en cours : ainsi, les processus examine et watch sont vus en cours de déroulement, plus exactement sont captés par le centre focal à un moment de leur déroulement. Il en est de même pour l'exemple (17) où l'on s'extirpe des pensées du centre focal en même temps que lui pour revenir à la situation en cours was inquiring. On remarque pourtant ici que le verbe inquire, verbe de parole, pourrait plus facilement être attendu au prétérit simple - c'est d'ailleurs le temps choisi pour la réponse he said. L'ajout de BE+-ING permet donc de renforcer la subjectivité du récit.

Que BE+V-ING aspectuel soit présent dans la nouvelle ne nous étonnera pas ; mais qu'en est-il du texte de théatre ? Pour rappel, la simple forme V-ING est récurrente dans les didascalies et alterne avec la forme au présent simple, à la façon d'un récit :

(18) SHERIFF [Chuckling.] Married to the law. [Moves toward the other room.] (34)

Dans l'exemple (18), le procès chuckle pourrait certes être montré en "cours de déroulement", issu d'un regard singulier, et donc être la réduction de "who is chuckling»; ce n'est pas le choix qui a été fait et comme la plupart du temps, ces procès duratifs/temporaires incidents à un support nominal et indexés à une situation $^{22}$ sont à la forme participiale, comme le sont aussi les procès ponctuels. Ils dictent les jeux d'acteurs, peuvent être interprétés sémantiquement comme des circonstances conditionnant les dialogues, tout comme une proposition participiale énonce une circonstance de l'événement principal.

Selon le postulat que le texte de théâtre devrait être objectif, il devrait donc être exempt de toute forme aspectuelle traduisant le regard singulier d'un énonciateur. Pourtant, on y trouve trois occurrences de BE+V-ING :

(19) HALE: [...] So I knocked again, and I thought I heard somebody say, 'Come in.' I wasn't sure, I'm not sure yet, but I opened the door-this door (indicating the door by which the two women are still standing) and there in that rocker- (pointing to it) sat Mrs Wright. (27) 
(20) MRS HALE [Who is standing by the table.] Well, I don't see any signs of anger around here [..] (30)

(21) MRS HALE rises, hands tight together, looking intensely at MRS PETERS, whose eyes make a slow turn, finally meeting MRS HALE's. A moment MRS HALE holds her, then her own eyes point the way to where the box is concealed. Suddenly MRS PETERS throws back quilt pieces and tries to put the box in the bag she is wearing. (34)

En (19), l'adverbe still associé à la position temporaire stand en singularise le regard; ce gros plan sur la position des femmes fait écho à la fin de la longue mise en place liminaire (exemple 13) dont nous reproduisons ici la dernière phrase :

(22) The women [...] stand close together near the door.

En (22), l'on plante un décor et logiquement le présent simple est convoqué, alors qu'en (19), l'adverbe still semble apporter la vision d'un œil particulier, celui qui remarque ou veut faire remarquer l'extension du temps, la longueur de cette immobilité et de ce silence encore inachevés, pris avant leur terme. Dès lors, sans être le regard spécifique d'un seul observateur, cette indication scénique informant sur le jeu d'acteur et la position des personnages doit logiquement prendre vie dans l'œil du spectateur lambda; et cela sera effectif grâce au verbe indicating qui amorce la didascalie et permettra à l'acteur de faire un geste en direction de ces femmes, et mettre le focus sur le fait qu'elles sont les seuls personnages à n'avoir pas encore bougé : on note d'ailleurs de la relative by which the two women are still standing qu'elle est continuative et permet de poser le repère la porte pour aller jusqu'à l'item les femmes.

En (20), la forme apparaît bel et bien accolée au nom dans une relative non réduite : nous remarquons qu'elle signale également que la position est temporaire, mais pas seulement. On aurait pu avoir: Mrs HALE [standing by the table], pour montrer la position. L'ajout de la forme complète est donc voulu afin de souligner l'importance de la vision focalisante, de faire un gros plan sur la position du personnage qui a, devant elle, les indices qu'elle recherche et qu'elle n'interprète pas encore (I don't see any signs of anger here). La forme permet la saillance.

Dans l'exemple (21), qui ressemble à un passage narratif au présent de reportage, she is wearing témoigne encore de ce regard particulier que l'on pose, ici sur ce sac qui, potentiellement, va servir à cacher la preuve de la culpabilité de Minnie Wright. Toute la scène tourne autour du jeu de regard des deux protagonistes; on peut imaginer que, logiquement, les personnages et le public prennent pour cible visuelle ce sac comme source potentielle de résolution de l'action. Mais Mrs Peters va-t-elle aller jusque-là?

La forme BE+V-ING, via son lien à l'aspect, signale un regard, une vision focalisante qui doit concerner tout observateur dans sa saisie des événements, à la façon d'une indication scénique destinée à l'expérienceur.

\section{Conclusion}

Le croisement du genre d'investigation et des champs génériques narratif et théâtral, qui ont une structure et un système énonciatif propres, induit des similarités et des différences dans le traitement du regard. Si les éléments lexicaux clés du genre d'investigation que sont les verbes LOOK, SEE et KNOW s'insèrent logiquement dans les formes théâtrales et narratives, la forme BE+V-ING, témoin d'un regard particulier, est logiquement plus rare dans les didascalies que la nouvelle. Au-delà de ces évidences, c'est l'utilisation subvertie de cette thématique du regard, à des fins de guidage, de 
caractérisation et même plus de critique, qui varie en fonction du champ générique, comme nous avons tenté de le démontrer. La pièce tend à l'objectivité, la nouvelle s'affirme comme très subjective. Mais si différentes ces stratégies semblent être, elles convergent pourtant vers le même point: une technique "narrative" mimétique, au plus près des personnages, sans commentaires de la part de l'instance narratrice : les deux formats courts, sans coupure en chapitre ou en acte comme dans la pièce ou le roman, et l'utilisation massive du discours direct pour un suivi « en temps réel », dans le temps des personnages, permettent au narrateur de se désengager, de se distancier ou de feindre de se distancier - du monde représenté pour laisser le lecteur/ spectateur se positionner. Ce mimétisme, conventionnel pour le théâtre, est permis, dans la nouvelle, par la focalisation centrée sur le personnage principal, technique narrative classique pour les récits modernistes de l'époque, et qui s'adapte parfaitement au genre de l'investigation; la vision "avec » permet la découverte pas à pas, en même temps que le détective. Néanmoins, le côté "problématisation" et "questionnement" présent dans la pièce est moins saillant dans la nouvelle qui assène plus qu'elle ne suggère, vu qu'elle prend le point de vue du personnage de la pièce le plus affirmé. Dans tous les cas, l'importance du regard, du point de vue, est diégétiquement et linguistiquement revendiquée dans les deux œuvres: Susan Glaspell joue avec les atouts que lui offrent les deux champs génériques pour faire du genre d'investigation une «vitrine » du savoir féminin, «vitrine» qui pourra être vue et lue par divers lectorats et audiences, tant le croisement enquête, pièce en un acte et nouvelle peuvent toucher alors un public différent.

\section{BIBLIOGRAPHIE}

Sources primaires :

GLASPELL, S., 2010 [1916], Trifles dans L. Ben-Zvi and J.E Gainor (eds), Susan Glaspell: The Complete Plays, Jefferson, McFarland \& Company, Inc., p. 24-34.

GLASPELL, S., 2010 [1917], « A Jury of Her Peers » dans P. L. Bryan et M. C. Carpentier (eds.), Her America: "A Jury of Her Peers" and Other Stories by Susan Glaspell, Iowa City, University of Iowa Press, p. 81-102.

Sources secondaires :

BIBER, D., Conrad, S., 2009. Register, genre, and style, Cambridge, Cambridge University Press.

CHUQUET, J., 2003, « Look et See: deux orientations différentes du repérage » dans J. Chuquet (éd), Verbes de Parole, de Pensée, de Perception. Etudes Syntaxiques et Sémantiques, Rennes, Presses Universitaires de Rennes, p.158-172.

CLAUSSON, N., 2001, « The Case of the Purloined Genre: Breaking the Codes in Susan Glaspell's 'A Jury Of Her Peers' ", Genre: Forms of Discourse and Culture, Spring-Summer 34, University of Oklahoma, Duke University press, p. 81-100 
DENDALE, P. , TASMOWSKI L, 1994. « Présentation. L'évidentialité ou le marquage des sources du savoir ", Langue française, $\mathrm{n}^{\circ} 102$. Les sources du savoir et leurs marques linguistiques. p. 3-7.

EVRARD, F., 1996, Lire le roman policier, Paris, Dunod.

GAINOR, E.J., 2001, Susan Glaspell in Context; American Theater, Culture, and Politics, 1915-48, Ann Arbor, University of Michigan Press.

GENETTE, G., 2007 [1972, 1983], Discours du récit. Essai de méthode, Paris, Editions du Seuil.

GOYET, F. ,1993, La nouvelle 1870-1925, Paris, PUF.

HAMBURGER, K., 1986, Logique des genres littéraires, Paris, Seuil.

JUNG, U., 1994, L'énonciation au théâtre. Une approche pragmatique de l'autotexte théâtral. Tübingen, Narr.

KERBRAT-ORECCHIONI, C., 1980, L'énonciation: de la subjectivité dans le langage, Paris, Armand Collin.

LARREYA, P., 1999, «BE + -ING est-il un marqueur d'aspect ? », Anglophonia/Sigma 03

< http://journals.openedition.org/anglophonia/682>

LINTVELT, J., 1981. Essai de typologie narrative : le " point de vue ». Théorie et analyse, Paris, Corti.

MALRIEU, D., RASTIER, F., 2001, « Genre et variation morphosyntaxique », Traitement Automatique des langues, vol. 42, $\mathrm{n}^{\circ} 2$, Lavoisier, Cachan, p. 548-577.

MELAC, E., 2014. L'évidentialité en anglais - approche contrastive à partir d'un corpus anglais-tibétain. Université de la Sorbonne nouvelle - Paris III. Français. <NNT : 2014PA030172><tel-01230545>

MILLER, P., 2003. « La complémentation directe et indirecte de verbes de perception en anglais » dans J. PAUCHARD (dir) Les prépositions dans la rection verbale (domaine anglais), Presses Universitaires de Reims, p 115-135.

$<$ https://docs.google.com/viewer?

$\mathrm{a}=\mathrm{v} \&$ pid=sites\&srcid=ZGVmYXVsdGRvbWFpbnxwaGlsaXBtaWxsZXJsa

W5ndWlzdGljc3xneDo2NjJmMTY5YTMwMTJjNmEz>

PETITJEAN, A., 2009, “Textes dramatiques et science du langage” dans C. Despierres et al. (eds), La lettre et la scène : linguistique du texte de théâtre, Dijon, Éditions universitaires de Dijon, p.27-41.

PRUNIER, M., 2001, L'analyse du texte de théâtre, Paris, Armand Collin.

RULLIER THEURET, F., 2003, Le texte de théâtre, Paris, Hachette supérieur.

TEINTURIER, F., 2008, Heinrich Mann et la nouvelle. Pratique d'un genre entre roman et théâtre, Peter Lang, Berne.

\section{NOTES}

1. Ce terme anglais désigne des choses sans importance, des vétilles.

2. Nous adopterons la terminologie « arborescente » de Malrieu et Rastier (2001:549) tout en la simplifiant: "champ générique » pour le théâtre et le récit en ce qu'ils regroupent d'autres genres discursifs, " genre discursif " pour la nouvelle ou le roman qui entrent traditionnellement dans le champ générique "récit». En ce qui concerne l'enquête, l'histoire d'investigation, elle représente, selon ce schéma, un «sous-genre » du roman. Bien consciente des raisons de cette appellation dans la logique arborescente du schéma, nous nous contenterons de répéter le mot 
"genre» en ce qui concerne l'histoire d'investigation pour souligner les conventions thématiques et linguistiques qui la caractérisent. Ce «sous-genre» tel qu'il apparait d'ailleurs chez Malrieu et Rastier est à relier dans notre corpus non pas au roman, mais à la nouvelle et à la pièce de théâtre, ce que n'envisage pas le schéma. Nous n'avons pas la conviction que l'histoire d'investigation constitue aux Etats-Unis, au début du 20eme siècle, un véritable « sous-genre » de la nouvelle, et il semble certain que ce ne soit pas un sous-genre de la pièce de théâtre.

3. Nous soulignons; dans cet article, toutes les mises en gras seront de notre fait.

4. Eléments d'informations tirés du premier chapitre de Gainor 2001 intitulé « The One-Act Play in America ».

5. Goyet 1993, $61 \mathrm{ff}$.

6. Goyet 1993 rappelle que l'intérêt de la nouvelle est « ailleurs que dans le personnage » (66) et affirme, à la suite de Todorov, que le personnage de la nouvelle n'a pas de «profondeur psychologique » (67). Le roman, dit-elle encore, «construit ses personnages au lieu de les faire surgir tout armés dans l'esprit du lecteur en recourant à des types » (85).

7. Teinturier (2008: 334) affirme que la nouvelle est bien la sœur du drame, selon la formule allemande de Storm. Il y voit deux stratégies esthétiques «effectivement identiques »: une « logique interne affirmée » et « une recherche de l'effet ».

8. Genette (2007 : 90) définit ainsi la scène : « La scène, le plus souvent « dialoguée », [...] réalise conventionnellement l'égalité de temps entre récit et histoire ».

9. Notre traduction.

10. Dans «A Jury of her Peers ", qui comprend 8219 mots, la fréquence d'apparition de ces trois mots est de $1 / 51$. Dans certains passages, la fréquence monte à $1 / 10$. Dans Trifles, qui comporte 5272 mots, la fréquence est de $1 / 46$, avec des pics à $1 / 14$. C'est encore plus flagrant si l'on considère la totalité du champ lexical de la vision (look, see, examine, glance, eyes etc) où la fréquence totale monte à $1 / 41$ dans « A Jury of her Peers » et à $1 / 44$ dans Trifles.

11. Chuquet (2003:158) rappelle que «SEE pose d'emblée le vu».

12. Oxford Advanced Leaner's Dictionary, 7ème édition.

13. «[A]vec un objet indirect en AT, le mouvement est dirigé vers le référent de l'objet indirect, qui est une cible, mais ne l'atteint pas, ou en tous cas ne l'atteint pas pleinement. » Miller (2003 : 4)

14. Chuquet (2003 : 158) dit que " pour le sujet [de SEE], dire « ne pas voir X », c'est asserter la non-existence de $\mathrm{X}$ de son point de vue.»

15. Voir aussi Clausson (2001: 87) : "Glaspell adapts this convention to validate women's domestic knowledge as anything but trivial ».

16. Nous entendrons la notion de subjectivité au sens de Kerbrat-Orechionni (1980), à savoir l'implantation du sujet parlant dans le discours ; elle parle notamment des «lieux d'ancrage [...] de la subjectivité langagière » (37)

17. De même, le verbe «suppose ", typique de la formulation d'hypothèses, apparaît 8 fois dans les dialogues, et ce sont toujours les femmes qui l'emploient.

18. On citera ici Dendale (1994: 4) : «En signalant dans l'énoncé la façon dont il a obtenu l'information qui y est transmise, le locuteur offre à son interlocuteur la possibilité d'évaluer luimême le bienfondé ou la fiabilité de cette information » (nous soulignons).

19. Nous adopterons la terminologie de Lintvelt (1981:67) : «Dans le type narratif actoriel, c'est un des acteurs qui sert de centre d'orientation aux plans perceptif-

psychique, temporel, spatial et verbal ». La perspective actorielle est proche de ce que Genette (2007) appelle « focalisation interne ».

20. Cette omniscience se fait jour dans une didascalie plus surprenante que les autres qui fait état de la conscience de Mrs Peters : "With a sigh, is about to sit down in the rocking-chair. Before she is seated realizes what chair it is; with a slow look at it, steps back» (Trifles : 29). Cette indication dicte à 
nouveau le jeu d'acteur qui doit physiquement, visuellement, traduire cette prise de conscience pour le spectateur lambda.

21. Deux exceptions sont toutefois à noter : in the left rear corner of the room (Trifles: 29) et to the door at the right (Trifles: 29 ).

22. Nous renverrons le lecteur à l'article de Merle 2009, «Les constructions participiales en anglais », pour les notions d'incidence (415) et d'indexation (420).

\section{RÉSUMÉS}

Cet article propose d'étudier la manifestation linguistique du regard et du point de vue dans deux œuvres de Susan Glaspell : Trifles (1916), pièce en un acte, et son adaptation en nouvelle par le même auteur, «A Jury of her Peers" (1917). C'est l'entrelacs du genre d'investigation et des champs génériques narratif et théâtral qui, à la fois, provoque des similitudes dans le traitement du regard, mais aussi et surtout des différences : l'utilisation des verbes LOOK, SEE et SEEM d'une part, et la forme BE+V-ING d'autre part, feront l'objet d'une étude comparative puisque leur emploi semble conditionné par le genre discursif. Nous relierons ces phénomènes linguistiques à la stratégie narrative à l'œuvre dans les deux textes et à l'interprétation de la critique sociétale de Susan Glaspell.

This article focuses on the linguistic treatment of vision and point of view in Susan Glaspell's masterpieces Trifles (1916) and «A Jury of her Peers» (1917). They belong to different generic fields - respectively drama and narrative -, and meet another genre - detective fiction-, as they happen to deal with a crime scene. Intertwining these genres causes similarities but also (mainly) differences when dealing with vision and point of view: we will particularly study the occurrences of LOOK, SEE, and SEEM, and BE+V-ING in both works, and we will try to show their importance in terms of narrative strategy. They are of prime importance in Susan Glaspell's painting of her society.

\section{INDEX}

Mots-clés : Genre, point de vue, perception, récit, théâtre, Susan Glaspell

Keywords : Genre, viewpoint, perception, narrative, drama, Susan Glaspell

\section{AUTEUR}

\section{MARIE-PIERRE MAECHLING}

Université de Strasbourg, EA1339 LILPA, Fonctionnements Discursif et Traduction 